EPJ Web of Conferences 114,02028 (2016)

DOI: $10.1051 /$ epjconf/201611402028

(C) Owned by the authors, published by EDP Sciences, 2016

\title{
Effect of tip vortices on membrane vibration of flexible wings with different aspect ratios
}

\author{
Mustafa Serdar Genç ${ }^{1, a}$, Halil Hakan Açikel ${ }^{1}$, Hacımurat Demir ${ }^{1,2}$, Mustafa Özden ${ }^{1}$, Mücahit Çağdaş $^{1}$, Iliasbek Isabekov ${ }^{1}$ \\ ${ }^{1}$ Wind Engineering and Aerodynamic Research Group, Department of Energy Systems Engineering, Erciyes University, Kayseri, Turkey \\ ${ }^{2}$ Department Of Mechanical Engineering, Aksaray University, Aksaray, Turkey
}

\begin{abstract}
In this study, the effect of the aspect ratio on the aerodynamics characteristic of flexible membrane wings with different aspect ratios $(\mathrm{AR}=1$ and $\mathrm{AR}=3$ ) is experimentally investigated at Reynolds number of 25000. Time accurate measurements of membrane deformation using Digital Image Correlation system (DIC) is carried out while normal forces of the wing will be measured by helping a load-cell system and flow on the wing was visualized by means of smoke wire technic. The characteristics of high aspect ratio wings are shown to be affected by leading edge separation bubbles at low Reynolds number. It is concluded that the camber of membrane wing excites the separated shear layer and this situation increases the lift coefficient relatively more as compared to rigid wings. In membrane wings with low aspect ratio, unsteadiness included tip vortices and vortex shedding, and the combination of tip vortices and vortex shedding causes complex unsteady deformations of these membrane wings. The characteristic of high aspect ratio wings was shown to be affected by leading edge separation bubbles at low Reynolds numbers whereas the deformations of flexible wing with low aspect ratio affected by tip vortices and leading edge separation bubbles.
\end{abstract}

\section{Introduction}

Flexible membranes have been associated with a number of applications such as hang gliders, parachutes, paragliders, yacht sails, microlights, natural flyers such as bats and insects. Micro Air Vehicles (MAVs) and Unmanned Air Vehicles (UAVs), which are characterized by low flight speeds and small size, are of great interest to low Reynolds number applications. In low Reynolds number flow regime, viscous forces are higher than inertia forces. So the flow is separated from the wing and reattached over the wing, which is named as a laminar separation bubble. The flow separation is very important for MAVs or UAVs.

Gordnier and Attar [1] performed a numerical study on flexible membrane wings. They performed computations at $\operatorname{Re}=24300$ and angles of attack $\alpha=10^{\circ}$, $\alpha=16^{\circ}$ and $\alpha=23^{\circ}$ for wing with aspect ratio of 2. They noticed that there was a reasonable agreement between computational results with experimental PIV and surface deflection measurements. The flexible wing demonstrated a mean first mode structural response of increasing amplitude with increasing angle of attack. Because of favorable interactions between the flexible membrane wing and the unsteady flow over the wing, reduced separation and enhanced lift were obtained.

Tamai et al. [2] performed an experimental study in order to investigate the benefits of utilizing flexible membrane wings at low Reynolds numbers for MAV applications. Flow field measurements carried on different angle of attack for quantifying the transient behaviour of vortex and turbulent flow structures around the flexible membrane wings at by means of a highresolution Particle Image Velocimetry (PIV) system. They noticed that flexible membrane wings ensured better aerodynamic performance comparing their rigid counterpart at low Reynolds numbers. In addition, it was seen that the aerodynamic performance of the membrane airfoils were effected the flexibility (or rigidity) of membrane skins remarkably. The PIV measurements clarified that flexible membrane wings altered their camber automatically in order to accommodate incoming flows for balancing the pressure differences applied on the upper and lower surfaces of the wings at different angle of attack.

Béguin and Breitsamter [3] investigated effects of membrane pre-stress on the aerodynamic characteristics of elasto-flexible morphing wing for unmanned air vehicle applications. The results of force and deformation measurements conducted at several dynamic pressures elucidated that varying the pre-stress effectively allowed to influence the passive deformation of the wing surface and regulated the wing camber within a certain range at a given flow condition. Moreover, it was seen that the lift and drag characteristics were affected such that an improvement of the maximum lift-to-drag ratio between

\footnotetext{
$\overline{{ }^{a} \text { Corresponding author: musgenc@ }}$ erciyes.edu.tr
} 
$6 \%$ and $23 \%$ of the lowest values at a given flow condition could be achieved.

Genç [4] investigated unsteady aerodynamics and flow-induced vibrations of a low aspect ratio rectangular membrane wing with excess length. The experiments for the membrane and rigid wing models were conducted in a closed-loop open-jet wind tunnel with a circular working section of $760 \mathrm{~mm}$ in diameter at angles of attack ranging from $0^{\circ}$ to $25^{\circ}$ at Reynolds number of 48,700. Digital Image Correlation system was used for time-accurate measurements of membrane deformation. Furthermore, measurement of velocity field with Digital Particle Image Velocimetry system was conducted whereas normal forces of the wing were measured by means of a load-cell system. Obtained experimental results demonstrated that the camber of membrane wing with excess length excited the separated shear layer. Additionally, the membrane caused to generate a larger magnitude of the maximum camber in the both $2 \mathrm{D}$ and $3 \mathrm{D}$ rectangular membrane wings with excess length. On the other hand, dominant vibrational modes were obtained as a spanwise mode of two at the angles of attack of $6-10^{\circ}$ and $19-22^{\circ}$. On the other hand, dominant spanwise modes were three at 11 $18^{\circ}$ and $23-25^{\circ}$.

Rojratsirikul et al. [5] performed an experimental study of a low aspect ratio rectangular wing in a lowspeed, closed loop open-jet wind tunnel for a Reynolds number range of $2.4 \times 10^{4}-4.8 \times 10^{4}$. Time accurate measurements of membrane deformation were added with the flow field measurements. Analysis of the fluctuating deformation detected chordwise and spanwise modes, which were due to the shedding of leading-edge vortices as well as tip vortices. It was seen that the second mode in the chordwise direction became dominant as the vortex shedding took place at higher angles of attack. The dominant frequencies of the membrane vibrations were similar to those of two-dimensional membrane airfoils. Furthermore, measured vortex shedding frequency from the low aspect ratio rigid wing suggested that membrane vibrations occurred at the natural frequencies close to the harmonics of the wake instabilities. Additionally, it was seen that vortex shedding frequency from rigid wings showed significantly small effect of aspect ratio even when it was as low as unity.

Hefeng et al. [6] investigated four types of segmented flexible membrane airfoil which were based on NACA0012 numerically. They applied fluid-structure interaction method in the numerical simulation for the aerodynamic characteristics and response between flow field and structure of the segmented flexible airfoils. They noticed that the segmented flexible airfoils carried out a higher maximum lift coefficient, and postponed the stall effectively. Furthermore, the deformation of the flexible thin membrane decreased the scale of the separation vortex at the higher angles of attack. Threesegment flexible airfoil had the best aerodynamic characteristics among the four kinds of segmented forms of wings. It created a profit of $39 \%$ on lift when comparing with the traditional rigid airfoil.

Song et al. [7] studied on aerodynamics of rectangular membrane wings with low aspect ratio for Reynolds numbers in the range of $0.7-2.0 \times 10^{5}$. The lift and drag coefficients were measured for wings with varying aspect ratio, compliancy and pre-strain values. Linear elastic theory was utilized in order to predict the natural frequencies of membrane. It was seen from the measurements that the membrane wings had a higher lift slope, maximum lift coefficients and delayed stall.

$\mathrm{Hu}$ et al. [8] investigated the aerodynamic performances of flexible membrane wings in flapping flight experimentally. They compared two flexible membrane wings with different skin flexibility (i.e., flexible nylon wing and a very flexible latex wing) with a conventional rigid wing for the development of flapping wing Micro Air Vehicles (MAVs). The measurement results demonstrated that flapping motion would bring significant aerodynamic benefits when the flapping flight was in unsteady state regime, and the flexibility had better aerodynamic performances for both soaring and flapping flights. It was found that the flexible membrane wings had better overall aerodynamic performance over the rigid wing for soaring flight, especially for high speed soaring flight or at relatively high angle of attack.

Ke et al. [9] studied on analysis and flexible structural modeling for oscillating wing using aeroelasticity. Furthermore, they detailed the design principle, design process and range of application of such oscillating wing analytical method. A flexible structural oscillating wing model was set up, and relevant time response analysis and frequency response analysis were carried out. It was found from the analytical results that adopting the newtype driving way for the oscillating wing would not have flutter problems and would be able to produce propulsive force. Additionally, it would consume much less power than the fixed wing for generating the same lift.

Bleischwitz et al. [10] investigated the aeromechanics of membrane wings in ground effect. They conducted the experiments in wind tunnel with a moderate Reynolds number of $\mathrm{Re}=56,000$ utilizing rectangular rigid and membrane wings in the vicinity of the ground. In order to allow correlating and identifying instantaneous membrane deformation, time-resolved forces and membrane dynamics were captured in parallel. In order to resolve membrane motions, Digital Image Correlation (DIC) was utilized. Furthermore, Proper Orthogonal Decomposition (POD) was applied to study the spatiotemporal structure of membrane vibrations. Decomposition (POD) modes of membrane fluctuations were sufficient to capture $90 \%$ of all deformation energy closes to stall. In addition to this, spanwise structural modes were found by means of POD in regions of strong lift increment.

Attar et al. [11] studied on experimental characterization of aerodynamic behaviours of membrane wings in low Reynolds number of $\operatorname{Re}=13700,22600$ and 36300 and the angle of attack up to $27^{\circ}$. It was found that the pre-stall values were irresponsive to pre-strain level for the mean lift coefficient results. Furthermore, they noticed that the Reynolds number had a considerable effect on the pre-stall lift curve, with the lift-curve slope increasing with increasing Reynolds number for all the flexible cases and the rigid flat plate model. Reducing level of model pre-strain led to increase in the pre-stall 
drag coefficients generally. It was found that the flexiblemodel stall angles were similar to the rigid flat plate results with the exception of the highest Reynolds number. On the other hand, dynamic results for the flexible models appeared large RMS values of lift and drag at the largest Reynolds number, which mostly reduced with increasing angle of attack. The RMS values for the rigid flat plate were unremarkable comparing with the flexible models.

Wrist et al. [12] compared the aerodynamic properties for flexible membrane micro air vehicle (MAV) wings with flat and cambered frames. An Objet30 Pro 3D printer was used for construction of wing's frames. They tested the wings in a low speed wind tunnel at Reynolds number of 50000 with varying angle of attack from $-4^{\circ}$ to $24^{\circ}$. It was stated from the results that cambering the frames for the flexible MAV wings increased maximum lift and aerodynamic efficiency comparing with the flat frames. Furthermore, membrane wings with cambered frames resulted in more negative pitching moment than the flat frame, leading to the center of pressure located aft of the quarter-chord. An increase in frame camber caused to increase aero-induced membrane camber with regard to the adjusted chord line.

Viieru et al. [13] discussed recent progress in understanding the low Reynolds number unsteady fluid dynamics related to flapping wings, including pitching-up rotation, leading-edge vortices and wake-capturing mechanisms. They noticed that the structures of the leading-edge vortices changed with Reynolds number remarkably by investigating the flow structures at three Reynolds numbers, namely, 6000, 120 and 10. The leading edge vortex demonstrated an intense, conical structure, with a sustained spanwise flow at the vortex core, breaking down at nearly three-quarters of the span towards the tip at Reynolds number of 6000 . The vortex break-down was lost, and it was found that the leadingedge vortices connected to the tip vortices at Reynolds number of 120 . In addition to this, a vortex ring connecting the leading edge vortex, the tip vortex and the trailing vortex was observed at $\mathrm{Re}=10$.

Greenhalgh et al. [14] investigated aerodynamic properties of a two dimensional inextensible flexible airfoil. Measurement of the lift, drag and membrane tension forces acting on a single surface mylar membrane supported by elliptical rods at the leading and trailing edges were conducted in the experiments. The angle of attack at which separation occurs decreased with increasing excess length and the hysteresis range increased resulting in a narrow operating range for the highest excess lengths examined. They noticed that theoretical results were in a good agreement with experimental data for those angles of attack where the flow over the membrane was not separated. Furthermore, all of the membranes demonstrated maximum lift-to-drag ratios near zero angle of attack. They observed hysteresis of the membrane airfoil near zero angle of attack experimentally.

\section{Experimental Apparatus Measurement Methods}

The experiments were carried out in a low-speed, suction-type wind tunnel with a square working section of $500 \mathrm{~mm} \times 500 \mathrm{~mm}$ located at the Department of Energy Systems Engineering, University of Erciyes via Wind Engineering and Aerodynamics Research Group (WEAR) as shown in Figure 1. Turbulence intensity of the tunnel is $0.35 \%$ and the operating range of tunnel speed was 1-45 m/ s. In the experiments, two aspect ratios, $\mathrm{AR}=1$ and $\mathrm{AR}=3$ were considered. For membrane aerofoils, a membrane which was made from a black latex rubber sheet with a thickness of $t=0.2 \mathrm{~mm}$, Young's modulus of $E=2.2 \mathrm{MPa}$, and density of $\rho_{\mathrm{m}}=1$ $\mathrm{g} / \mathrm{cm}^{3}$ was used. The membrane was attached to the aerofoil-shaped leading and trailing edges for a membrane aerofoil. The wing used in the study was composed of a rigid stainless steel frame and attached membrane. This rigid frame was manufactured in order to have a cross-section of airfoil shape (as seen in Figure 2).

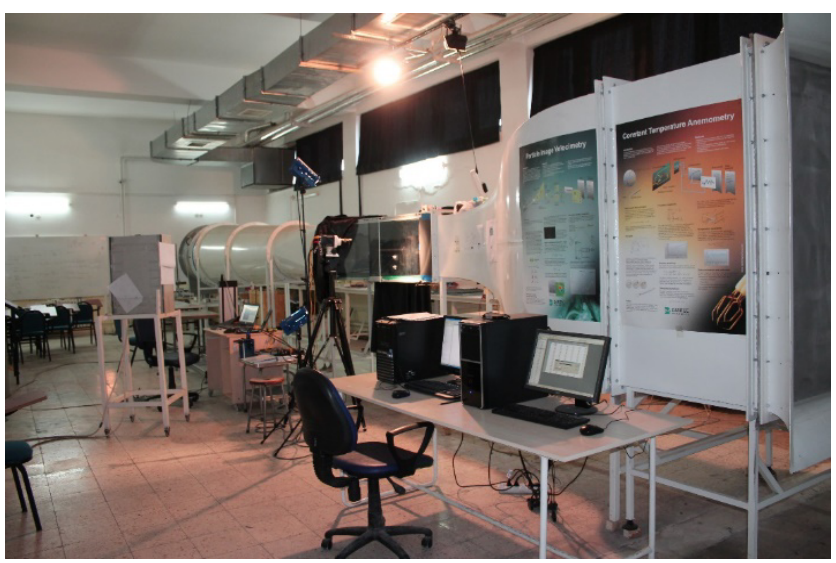

Figure 1. Photograph of the experimental set-up.

\subsection{Experimental Studies for Membrane Wing}

Details of experimental studies about the membrane wing are given below elaborately. First of all, the variations of aerodynamic force coefficients with respect to aspect ratio were investigated. After determination of the aerodynamic force coefficients, flow on the wing was visualized by means of smoke wire experiment.
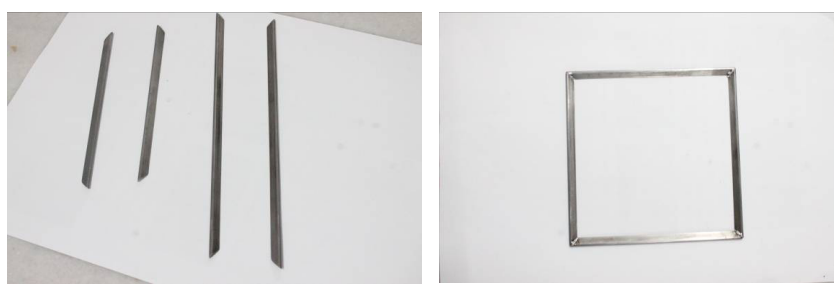


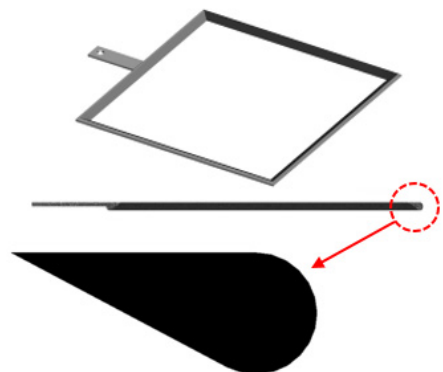

Figure 2. Schematic of the rectangular wing with cross-section of the rigid frame.

\subsubsection{Aerodynamics performance measurement}

Aerodynamic forces were measured at specific angles of attack from $0^{\circ}$ to $45^{\circ}$ by means of a computer controlled force measurement system having automatic change of angle. As seen from Figure 3, lift and drag forces are measured separately by means of strain gauges and these measured forces changed with coordinates according to the angle of attack of the wing. So, the measured values are converted to the lift $\left(\mathrm{C}_{\mathrm{L}}\right)$ and drag $\left(\mathrm{C}_{\mathrm{D}}\right)$ force coefficients in $\mathrm{x}$ and $\mathrm{y}$ axes by means of coordinate transformation.
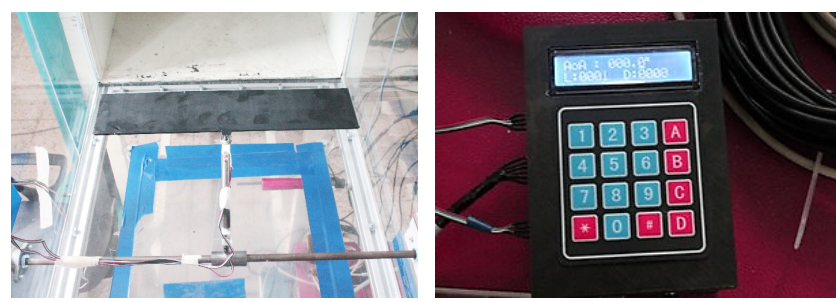

Figure 3. Computer controlled force measurement system.

\subsubsection{Deformation measurement}

Digital Image Correlation (DIC) system was used to measure the membrane deformations. The DIC system captures consecutive images and then calculates the displacement field over the specimen by following the deformation of spots (Figure 4) applied to the surface of the specimen using a cross-correlation method.

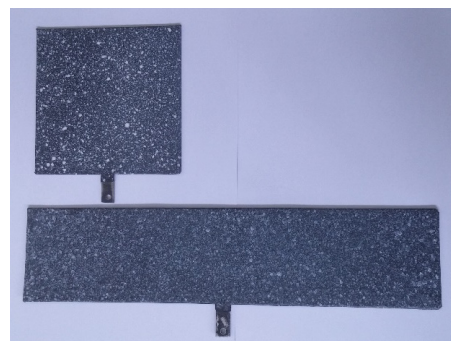

Figure 4. Flexible wings for deformation measurements.

\subsubsection{Flow visualization via smoke-wire}

Smoke-wire technique is preferred for the flow visualization due to the simplicity and reliability. A smoke-wire is utilized for heating the fluid and machine oil to mark the streamlines for flow visualization. Canon
EOS-D1100 camera was used for taking related experimental photographs. The main problem in recording the smoke lines is reflection of images in the plexiglas wall test section of the tunnel. For this reason, lighting and camera arrangements are carried out carefully to ensure dark medium as seen in Figure 5a. The expression of $\mathrm{z} / \mathrm{c}$ is utilized for the location of smoke wire as shown in Figure 5b. The behaviors of both laminar separation bubble and tip vortex are investigated in terms of various angles of attack, aspect ratios.

a)

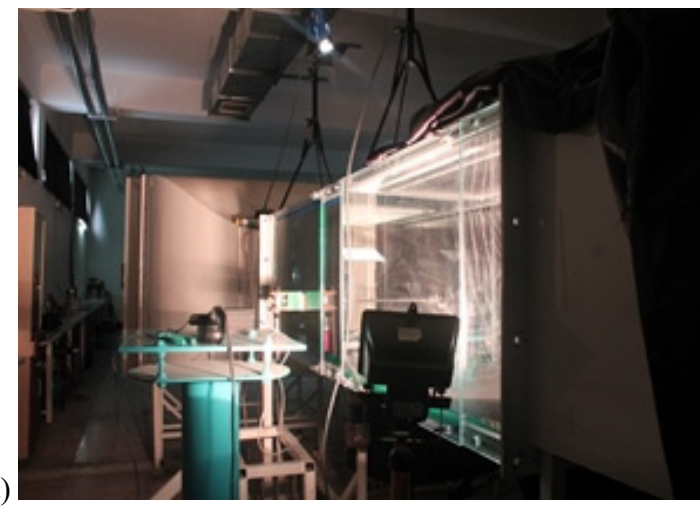

b)

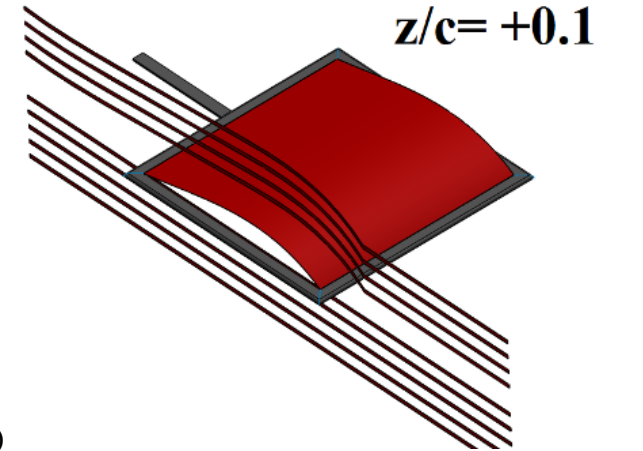

Figure 5. a) Dark medium for smoke-wire measurement b) Location of smoke wire on the membrane wing.

\section{Experimental Results}

\subsection{Determination of aerodynamic coefficients of flexible membrane wing}

The variation of $C_{L}$ and $C_{D}$ coefficients of membrane wing with respect to the angle of attack in various aspect ratios at $\mathrm{Re}=25000$ are given in Figure 6. According to the obtained results from these figures, stall angles of attack were $35^{\circ}$ and $19^{\circ}$ and the values of $C_{L \max }$ were 1.3 and 1.5 for $\mathrm{AR}=1$ and $\mathrm{AR}=3$, respectively. As the aspect ratio increased, stall angle and maximum lift decreased. 


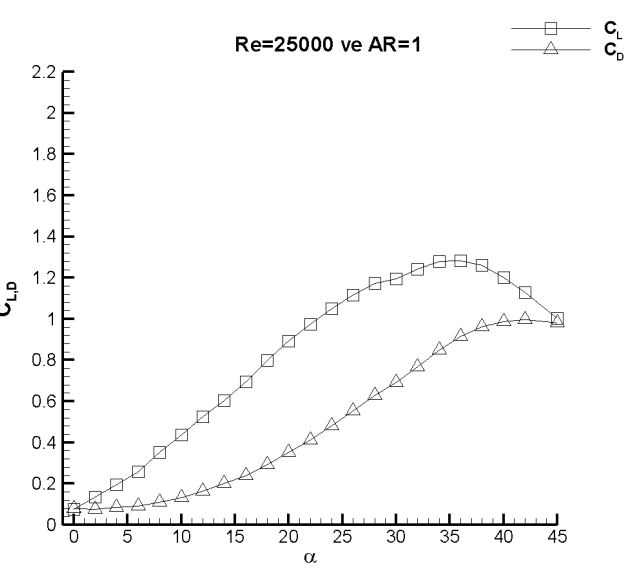

(a)

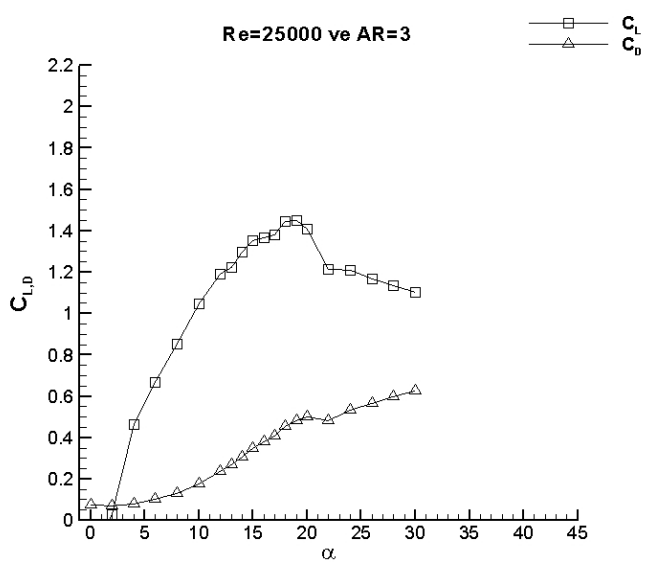

(b)

Figure 6. Aerodynamic force coefficients at various Re numbers.

\subsection{Deformation results}

Figure 7 and Figure 8 shows instantaneous membrane deformations for the wing with $\mathrm{AR}=1$ and $\mathrm{AR}=3$ at AoA $=12^{\circ}$. It is seen that the membrane deformations for the wing with $\mathrm{AR}=1$ change with time whereas for the wing with $\mathrm{AR}=3$ it has not instantaneous variations. Over the wing with $\mathrm{AR}=1$, tip vortices and leading edge separations are dominant, thus the flow over the wing are unsteady and the membrane deformations change instantaneously.

\subsection{Smoke-wire visualization results}

The variations of tip vortices with respect to the angle of attack are given from Figure 9 and Figure 10. Increase in angles of attack from $8^{\circ}$ to $20^{\circ}$ leads to improve tip vortices throughout the wake region at $\mathrm{Re}=25000$ and $\mathrm{AR}=1$ and $\mathrm{AR}=3$. These laminar separation bubbles moved towards to leading-edge and their size became smaller as angle of attack increased. Tip vortices delays stall for the wing at low aspect ratio, especially at low Re numbers due to affecting flow on the wing and separation bubble as seen in Figure 11 and 12. Separation bubble located near trailing-edge was occurred at low angle of attack.

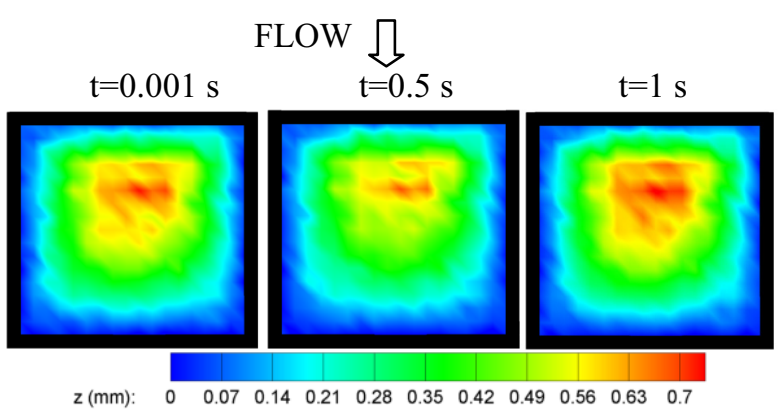

Figure 7. Instantaneous membrane deformations for the wing with $\mathrm{AR}=1$ at $\mathrm{AoA}=12^{\circ}$.

$$
\text { FLOW }
$$

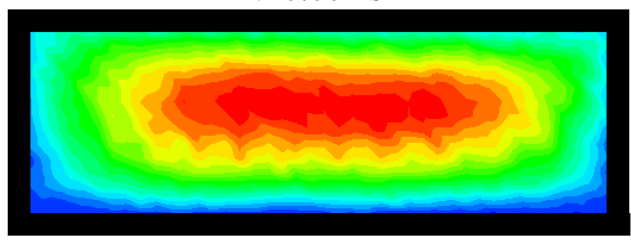

$\mathrm{t}=0.5 \mathrm{~s}$

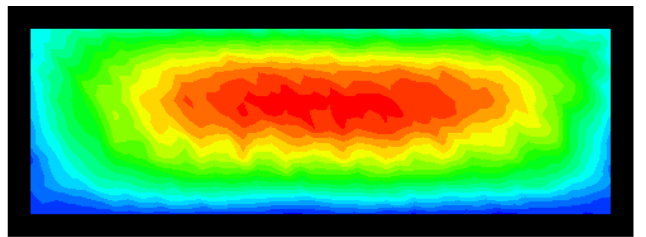

$\mathrm{t}=1 \mathrm{~s}$

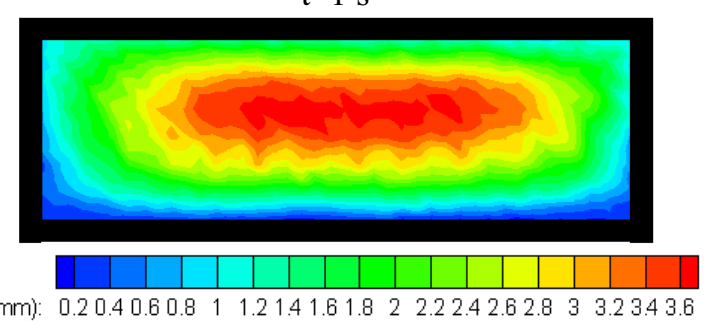

Figure 8. Instantaneous membrane deformations for the wing with $\mathrm{AR}=3$ at $\mathrm{AoA}=12^{\circ}$.

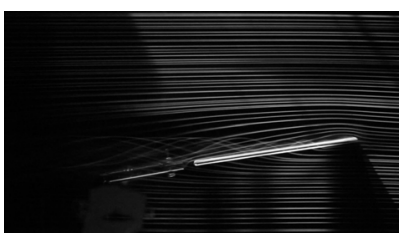

a) $\mathrm{AoA}=8^{\circ}$

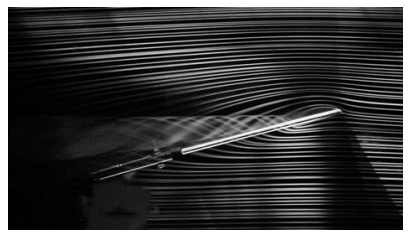

e) $\mathrm{AoA}=14^{\circ}$

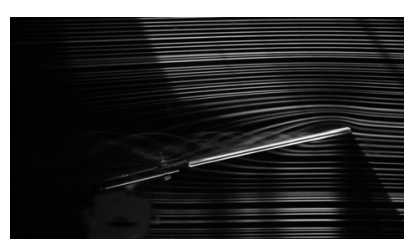

b) $\mathrm{AoA}=12^{\circ}$

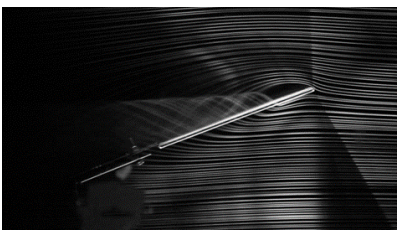

f) $\mathrm{AoA}=20^{\circ}$
Figure 9. Smoke-wire visualization for tip vortices at $\mathrm{z} / \mathrm{c}=$ $+0.1(\operatorname{Re}=25000$ and $\mathrm{AR}=1)$. 


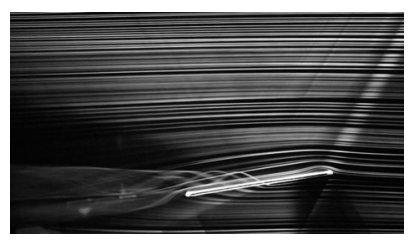

a) $\mathrm{AoA}=8^{\circ}$

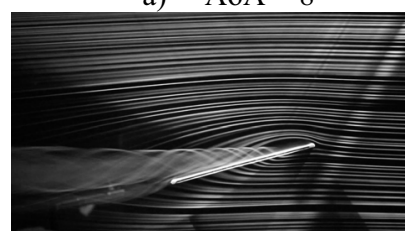

c) $\mathrm{AoA}=14^{\circ}$

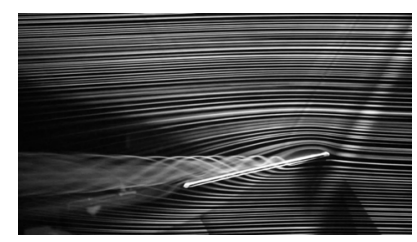

b) $\mathrm{AoA}=12^{\circ}$

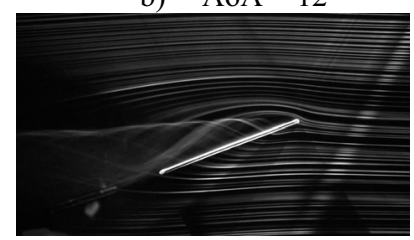

d) $\mathrm{AoA}=20^{\circ}$
Figure 10. Smoke-wire visualization for tip vortices at $\mathrm{z} / \mathrm{c}=$ $+0.1(\operatorname{Re}=25000$ and $\mathrm{AR}=3)$.

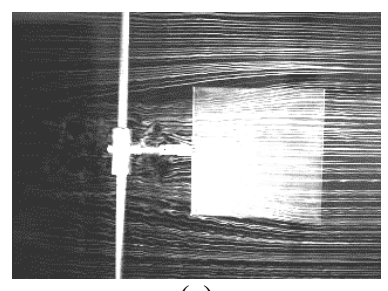

(a)

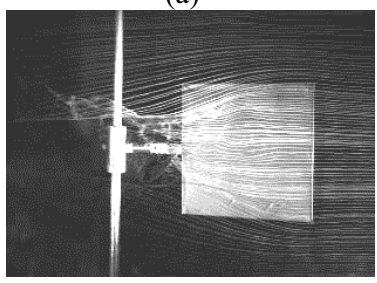

(c)

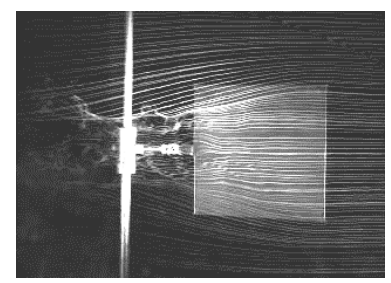

(b)

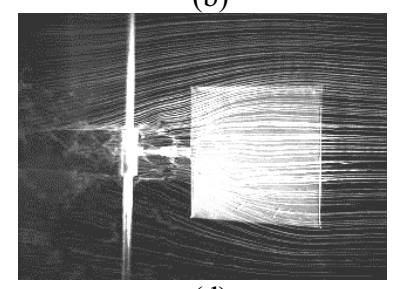

(d)
Figure 11. Top view smoke-wire visualization for tip vortices at $\mathrm{z} / \mathrm{c}=+0.1(\operatorname{Re}=25000$ and $\mathrm{AR}=1)$ for $(\mathrm{a}) \alpha=8^{\circ}$, (b) $\alpha=$ $16^{\circ}$, (c) $\alpha=20^{\circ}$ and (d) $\alpha=24^{\circ}$.

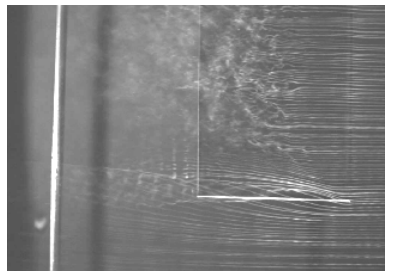

(a)

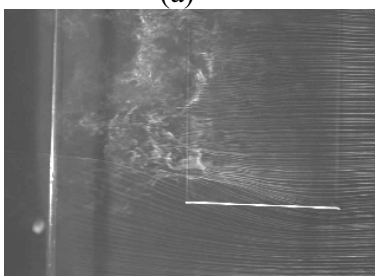

(c)

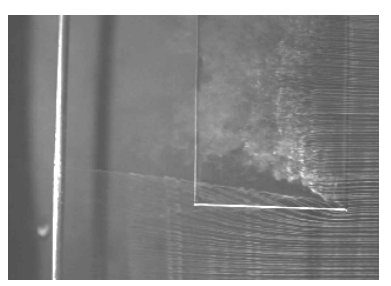

(b)

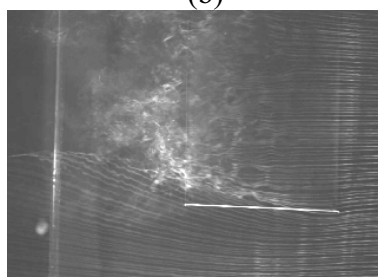

(d)
Figure 12. Top view smoke-wire visualization for tip vortices at $\mathrm{z} / \mathrm{c}=+0.1(\mathrm{Re}=25000$ and $\mathrm{AR}=3)$ for (a) $\alpha=8^{\circ}$, (b) $\alpha=$ $12^{\circ}$, (c) $\alpha=16^{\circ}$ and (d) $\alpha=20^{\circ}$.

\section{Conclusion}

The effect of tip vortices on membrane vibration of flexible wings with $\mathrm{AR}=1$ and $\mathrm{AR}=3$ was experimentally investigated at $\mathrm{Re}=25000$ in a lowspeed, suction-type wind tunnel. In the force measurements, it was obtained that the stall angles of attack were $35^{\circ}$ and $19^{\circ}$ and the values of $C_{\text {Lmax }}$ were 1.3 and 1.5 for $\mathrm{AR}=1$ and $\mathrm{AR}=3$, respectively. As the aspect ratio increased, stall angle and maximum lift decreased. In the DIC results, over the wing with $\mathrm{AR}=1$, tip vortices and leading edge separations are dominant, thus the flow over the wing are unsteady and the membrane deformations change instantaneously. In the flow visualization experiments showed that tip vortices delayed stall for the wing at low aspect ratio, especially at low Re numbers due to affecting flow on the wing and separation.

\section{Acknowledgments}

The authors would like to acknowledge funding from the Scientific and Technological Research Council of Turkey (TÜBİTAK) under the project no: 213M329, the Scientific Research Projects Unit of Erciyes University under the contract no: FDK-2015-6171, FBG-2014-5337 and FDA-2014-5273.

\section{References}

1. R.E. Gordnier, P.J. Attar, Impact of flexibility on the aerodynamics of an aspect ratio two membrane wing. $J$. Fluids Struct. 45, 138-152 (2014)

2. M. Tamai, J. Murphy, H. Hu, An experimental study of flexible membrane airfoils at low Reynolds numbers. AIAA Aerospace Sciences Meeting and Exhibit. 7-10 (2008)

3. B. Béguin, C. Breitsamter, Effects of membrane prestress on the aerodynamic characteristics of an elastoflexible morphing wing. Aerosp. Sci. Technol. 37, 138150 (2014)

4. M.S. Genç, Unsteady aerodynamics and flow-induced vibrations of a low aspect ratio rectangular membrane wing with excess length. Exp. Therm Fluid Sci. 44, 749759 (2013)

5. P. Rojratsirikul, M.S. Genc, Z. Wang, I. Gursul, Flowinduced vibrations of low aspect ratio rectangular membrane wings. J Fluid Struct. 27, 1296-1309 (2011)

6. D. Hefeng, W. Chenxi, L. Shaobin, S.X. Zhen, Numerical Research on Segmented Flexible Airfoils Considering Fluid-structure Interaction. Procedia Eng. 99, 57-66 (2015)

7. A. Song, X. Tian, E. Israeli, R. Galvao, K. Bishop, S. Swartz, K. Breuer, Aeromechanics of membrane wings with implications for animal flight. AIAA journal. 46(8), 2096-2106 (2008)

8. H. Hu, A.G. Kumar, G. Abate, R. Albertani, An experimental investigation on the aerodynamic performances of flexible membrane wings in flapping flight. Aerosp. Sci. Technol. 14(8), 575-586 (2010)

9. S. Ke, W. Zhigang, Y. Chao, Analysis and flexible structural modeling for oscillating wing utilizing aeroelasticity. Chin. J. Aeronaut. 21(5), 402-410 (2008)

10. R. Bleischwitz, R. Kat, B. Ganapathisubramani, Aeromechanics of Membrane Wings in Ground-Effect. 45th AIAA Fluid Dynamics Conf. 2764, (2015) 
11. P.J. Attar, B. J. Morris, W. A. Romberg, J. W. Johnston, R. N. Parthasarathy, Experimental Characterization of Aerodynamic Behavior of Membrane Wings in Low-Reynolds-Number Flow. AIAA journal. 50(7), 1525-1537 (2012)

12.A. Wrist, D. Pepley, Z. Zhang, J.P. Hubner, Aerodynamic Comparison of Flat and Cambered Frames for Flexible MAV Wings. 53rd AIAA Aerospace Sciences Meeting. (2015)

13. D. Viieru, J. Tang, Y. Lian, H. Liu, W. Shyy, Flapping and flexible wing aerodynamics of low Reynolds number flight vehicles. AIAA paper. 503, (2006)

14. S. Greenhalgh, H.C. Curtiss, B. Smith, Aerodynamic properties of a two-dimensional inextensible flexible airfoil. AIAA journal. 22(7), 865-870 (1984) 